\title{
General Anesthesia Increased the Risk of Atrial Fibrillation and Acute Kidney Injury in Transcatheter Aortic Valve Replacement
}

\author{
Ying Liang, MD,${ }^{1}$ Wei Wang, MD,${ }^{2} \mathrm{Xu}$ Wang, $M D,{ }^{2}$ Mingzheng Liu, MD,${ }^{3}$ Feilong Hei, MD,${ }^{1}$ \\ Yulong Guan, $\mathrm{MD}^{1}$ \\ ${ }^{1}$ Department of Extracorporeal Circulation, Fuwai Hospital, Chinese Academy of Medical Sciences and Peking Union Medical \\ College, Beijing, P.R. China \\ ${ }^{2}$ Department of Structural Heart Center, Fuwai Hospital, Chinese Academy of Medical Sciences and Peking Union Medical College, \\ Beijing, P.R. China \\ ${ }^{3}$ Department of Anesthesia, Fuwai Hospital, Chinese Academy of Medical Sciences \& Peking Union Medical College, Beijing, P.R. China
}

\section{ABSTRACT}

Purpose: To examine key impacts of anesthesia on newonset atrial fibrillation (AF) and acute kidney injury (AKI) in transcatheter aortic valve replacement (TAVR).

Methods: All consecutive patients who underwent transfemoral, transapical, and transaortic TAVR in Fuwai Hospital from 2012 to 2018 were retrospectively analyzed and dichotomized into 2 groups: TAVR under conscious sedation (CS) and under general anesthesia (GA). The primary endpoint was a composite of all-cause mortality, stroke, $\mathrm{AF}$, permanent pacemaker implantation, myocardial infarction, heart failure, high-grade atrioventricular block, and AKI at 1 year. Binary logistic regression and adjusted multilevel logistic regression were performed to analyze the predictors of $\mathrm{AF}$ and $\mathrm{AKI}$.

Results: A total of 107 patients were under CS and 66 patients under GA. No significant difference was observed in the composite endpoint $(51.5 \%$ vs. $41.2 \%$, GA vs. CS, $P=$ $.182)$ and $\geq$ mild paravalvular leakage (36.4\% vs. $31.4 \%$, GA vs. CS, $P=.589)$ at 1 year. However, the GA group had a significantly higher rate of intensive care unit (ICU) admission ( $84.8 \%$ vs. $6.5 \%, P<.001)$, AKI $(28.8 \%$ vs. $14.0 \%, P=.018)$, new-onset $\mathrm{AF}(15.2 \%$ vs. $5.5 \%$ at 1 year, $P=.036)$. Multivariable analysis revealed GA to be the significant predictor of new-onset $\mathrm{AF}$ (odds ratio $3.237,95 \%$ confidence interval 1.059 to $9.894, P=.039$ ) and AKI (odds ratio $2.517,95 \%$ confidence interval 1.013 to $6.250, P=.047)$.

Conclusion: GA was associated with higher rates of ICU admission, postoperative AKI, and new-onset AF. The results may provide new evidence that CS challenges universal GA.

Received September 29, 2020; accepted November 1, 2020.

Correspondence: Yulong Guan MD, Department of Extracorporeal Circulation, Fuwai Hospital, Chinese Academy of Medical Sciences \& Peking Union Medical College, 167 Beilishi Road, Xicheng district, Beijing 100037, P. R. China; (86)010-88396403; (e-mail: guanyulong@yahoo.com). Feilong Hei MD, Department of Extracorporeal Circulation, Fuwai Hospital, Chinese Academy of Medical Sciences \& Peking Union Medical College, 167 Beilishi Road, Xicheng district, Beijing 100037, P. R. China; (e-mail: heifeilong@126.com).

\section{INTRODUCTION}

Although the first transcatheter aortic valve replacement (TAVR) was undertaken under mild sedation and local anesthesia (LA), general anesthesia (GA) was adopted as the standard at some institutions for these high-acuity patients [Leon 2010; Leon 2016]. Now there is a trend toward the more liberal use of LA or conscious sedation (CS) for TAVR. According to the Transcatheter Valve Therapy (TVT) registry, from April 2014 to June 2015, the percentage of national TAVR cases performed under CS rose from $11 \%$ per quarter to $20 \%$ per quarter [Hyman 2017], and from 2016 to 2019, the proportion increased from $33.4 \%$ to $64.1 \%$ [Butala 2020]. Although there were exclusively limited data published in this regard, the vast majority of the literature confirmed the safety and effectiveness of CS in TAVR, and recently, this was approved by a randomized trial [Thiele 2020]. Previous studies have described that the use of CS correlated with improved outcomes compared with GA, including decreased in-hospital or 30-day mortality, lower expenditures of health care resources, briefer intensive care unit (ICU) and hospital length of stay, and more recently, reduced risk of postoperative delirium [Attizzani 2015; Attizzani 2019; Hyman 2017; Maier 2020 Marcantuono 2015; Mosleh 2019].

Nevertheless, GA is deemed to provide more stable conditions by preventing patients from moving and a quick conversion to bail-out surgery in case of procedural complications [Konigstein 2017]. Moreover, new devices and improvements in existing devices have reduced procedural complications [Hellhammer 2018; Manoharan 2018; Van Mieghem 2012]. On the other hand, mortality solely attributable to GA ranged from 34 per million to 357 per million [Bainbridge 2012]. From these points, the risk related to GA might outweigh its benefits. In the literature, the composite endpoint of anesthetic strategies is unclear; in particular, the impact of anesthesia strategies on acute kidney injury (AKI) and newonset atrial fibrillation (AF) is lacking.

Accordingly, the objective of this study was to explore the status of different anesthetic strategies in TAVR and analyze the impacts of anesthetic strategies on clinical outcomes in a single center. 


\section{METHODS}

We did a retrospective chart review of consecutive cases assigned for TAVR from September 2012 to January 2018 and finally enrolled the patients who underwent TAVR successfully via 3 major approaches (transfemoral [TF], transapical [TA], and transaortic [TAO] routes). Patients with an aborted procedure and those requiring a surgical conversion were excluded. Multidetector computed tomography (MDCT), fluoroscopy, transesophageal echocardiography (TEE), and transthoracic echocardiography (TTE) were used to ensure the best prosthesis-patient match, to assess valve position and function after deployment, and to identify immediate complications. Two different strategies of anesthesia were used during the operation, including traditional GA and recent CS anesthesia. After the procedure, patients were transferred to the ward or ICU in consideration of baseline characteristics, periprocedural details, and anesthetic strategies.

\section{Anesthetic Strategies}

TAVR via TAO approach or TA approach was implemented routinely under GA. In regard to TF-TAVR, the anesthesia type was selected on a case-by-case basis, primarily by the cardiac anesthesiologists. Patients who are restless would be considered with GA. Patients who were deemed in poor general condition by the anesthesiologists would be planned with CS. In addition, if the procedure was conducted by operators with deficient experience, GA would be performed preemptively to avoid underlying emergencies.

Induction in the GA group was achieved using intravenous sufentanil (1 to $2 \mu \mathrm{g} / \mathrm{kg})$, cisatracurium $(0.15 \mathrm{mg} / \mathrm{kg})$, and etomidate $(0.3 \mathrm{mg} / \mathrm{kg})$. A supplemental dose of propofol $(0.5$ to $1 \mathrm{mg} / \mathrm{kg}$ ) was administrated if the deep sedation was not reached. Anesthesia was maintained with $0.5 \%$ to $1 \%$ sevoflurane combined with intravenous propofol of 1 to $2 \mathrm{mg} / \mathrm{kg} / \mathrm{h}$. Invasive blood pressure monitoring was completed via radial artery, and a pacing Swan-Ganz catheter was installed via the right jugular vein by the anesthesiologist. In the CS group, every patient received propofol $(0.5$ to $1.0 \mathrm{mg} / \mathrm{kg})$, midazolam $(0.02$ to $0.05 \mathrm{mg} / \mathrm{kg}$ ), and sufentanil ( 3 to $5 \mu \mathrm{g}$ ) for induction of $\mathrm{CS}$, and invasive lines are inserted in the same way as for GA. During maintenance of sedation, patients received 1 to $2 \mathrm{mg} /$ $\mathrm{kg} / \mathrm{h}$ of propofol and $0.5 \mu \mathrm{g} / \mathrm{kg} / \mathrm{h}$ of dexmedetomidine. Infusion doses of medication were adjusted up or down at the discretion of the anesthesiologists to maintain optimal sedation. A bolus dose of unfractionated heparin (100 IU/kg) was administered for all of the cases with a goal activated clotting time of $>250 \mathrm{~s}$.

\section{Data Acquisition}

Patient data were collected by 2 of the authors using electronic medical records. Information was also gathered over the telephone using a predefined questionnaire.

\section{ENDPOINTS}

The primary endpoint was a composite of all-cause mortality, stroke, new-onset AF, permanent pacemaker implantation
(PPI), myocardial infarction, heart failure, high-grade atrioventricular block, and AKI at 1 year. All endpoints were defined in accordance with Valve Academic Research Consortium 2 (VARC-2) criteria [Kappetein 2012]. Secondary endpoints included major vascular complication, paravalvular leakage (PVL), readmission, and thrombosis. Operative duration was defined as first incision to last suture, and anesthetic duration was measured from induction to leaving the operation room. Readmission was determined as readmission events that were relevant to surgery or heart disease.

To explore the impact of different approaches on the results, patients in the GA group were divided into 3 subgroups: TF, TAO, and TA subgroups. Additionally, all TF-TAVR patients were subdivided into 2 subgroups to verify the effect of different strategies on patients with same access. The ethics committee of Fuwai Hospital approved the retrospective collection of data, and the study complies with the Declaration of Helsinki. Informed consent was waived by the ethics committee.

\section{Statistics}

Categorical variables are expressed as percentages, and continuous variables as either mean \pm standard deviation (SD) or median (interquartile range). Continuous variables were analyzed using a t test or Mann-Whitney $U$ test, and categorical variables with the $\chi^{2}$ test or Fisher exact test. One-way analysis of variance (ANOVA) or Kruskal-Wallis were applied to test continuous variables in TF, TA, and TAO subgroups. Kaplan-Meier curves were used to estimate time-to-events, with the log-rank test to compare endpoints between groups. We ran a univariate binary logistic regression and subsequently an adjusted multilevel logistic regression to analyze the predictors of $\mathrm{AF}$ and $\mathrm{AKI}$. Variables that were significant $(P<.1)$ on univariate analysis or clinically relevant were included in a multivariate regression analysis to explore whether endpoints could have been biased by baseline differences. The details of univariate analyses and test of multicollinearity are shown in Supplemental Material (including Tables S4-S6. Variables chosen for inclusion in the model predicting AF were age, COPD, GA, reintervention, NYHA class $>$ II, peripheral vascular disease, diabetes, and TAO approach. Variables in the AKI predicting model included GA, age, diabetes, NYHA class $>$ II, GFR $>60 \mathrm{~mL} / \mathrm{min} / 1.73 \mathrm{~m}^{2}$, anesthetic duration, peripheral vascular disease, reintervention, ACEI/ARB drugs within $48 \mathrm{~h}$, TAO approach, and male. $P$ values for interaction were calculated for prespecified subgroup analyses to assess consistency of the treatment effect. Tests of hypotheses were 2 -sided. The confidence level was set at $95 \%$, and a $P$ value $<.05$ was deemed statistically significant. Statistical analysis was done using IBM SPSS Statistics for Windows, version 25.

\section{RESULTS}

We collected data from 201 patients assigned for TAVR via TF, TAO, and TA approach after reviewing the medical system. The rate of conversion to CS was $2.5 \%$ ( 5 of 201). There were 28 patients who were converted to open heart aortic surgery or exclusively balloon aortic valvuloplasty excluded. 
Table 1. Baseline Characteristics Based on the Entire Sample*

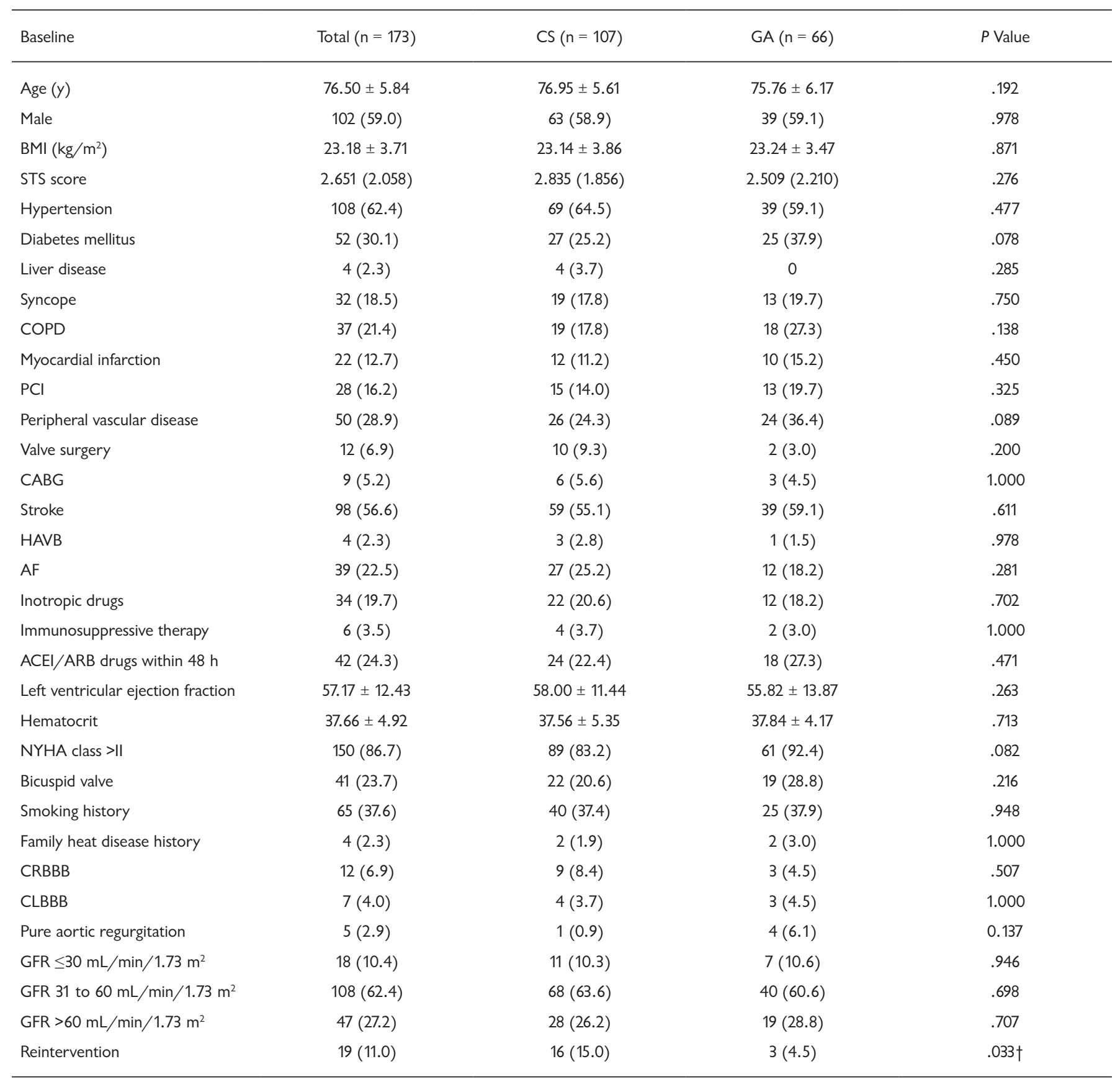

*Values are mean $\pm S D, n(\%)$, or median (interquartile range).

†Significant.

ACEl indicates angiotensin converting enzyme inhibitors; AF, atrial fibrillation; ARB, angiotensin receptor blocker; BMI, body mass index; CABG, coronaryartery bypass surgery; CLBBB, complete left bundle branch block; COPD, chronic obstructive pulmonary disease; CRBBB, complete right bundle branch block; CS, conscious sedation; GA, general anesthesia; GFR, glomerular filtration rate; HAVB, high-grade atrioventricular block; NYHA, New York Heart Association; $\mathrm{PCl}$, percutaneous coronary intervention; STS, Society of Thoracic Surgeons. 
A total of 173 patients were enrolled: 107 TAVR were under CS, and 66 under GA. In the GA group, 27 cases were performed via TF approach, 13 via TA approach, and 26 via TAO approach, whereas 107 patients in the CS cohort were conducted via TF access. There were in all 134 TF-TAVR based on the above summary. The median follow-up time was 29 (30) months, with the longest being 73 months.

Table 1 summarizes baseline characteristics between the CS cohort and GA cohort. Preoperative characteristics were similar between the 2 groups, with the exception who there was a significantly higher proportion of patients who received reintervention in the CS group $(15 \%$ vs. $4.5 \%$, CS vs. GA, $P=$ $.033)$. In the GA subgroups, there were statistical differences in the aspects of body mass index (BMI; $24.48 \pm 3.45,22.72 \pm$ 3.05 , and $21.84 \pm 3.75$ in TA, TF, and TAO, respectively; $P=$ $.046)$, bicuspid valve $(34.6 \%, 37.0 \%$, and $0 \% ; P=.037)$, pure aortic regurgitation $(15.4 \%, 0 \%$, and $0 \% ; P=.043)$, and GFR $>60 \mathrm{~mL} / \mathrm{min} / 1.73 \mathrm{~m} 2(46.2 \%, 14.8 \%$, and $23.1 \% ; P=.037)$, and no otherwise statistical difference was observed in basic information (Table S7). For $134 \mathrm{TF}$ TAVR, there were no statistically significant differences between the 2 subgroups, with the exception of diabetes (GA $51.9 \%$ vs. CS $25.2 \%$; $P=$ .007 ), peripheral vascular diseases (GA $48.1 \%$ vs. CA $24.3 \%$; $P=.015)$, and NYHA class $>$ II (GA $100 \%$ vs. CS $83.2 \% ; P=$ .048) (Table S8).

\section{Postoperative Results}

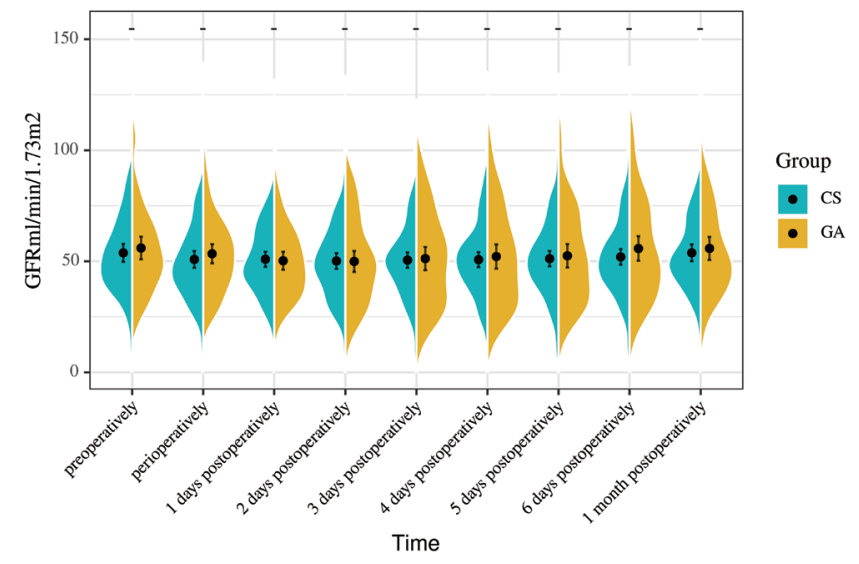

Figure 2. GFR over time. CS indicates conscious sedation; GA, general anesthesia; GFR, glomerular filtration rate.

GA was associated with a higher rate of admission to the ICU (84.8\%) compared with CS (6.5\%). In the GA subgroups, all patients with TA or TAO access were moved to the ICU, and $63 \%$ of patients under GA via TF access were transferred to the ICU. No significant differences between the GA and CS groups were observed in the aspects of operative duration [GA $120(88.75,146.25)$ vs. CS $115(100,150) ; P=.811]$, anesthetic duration [GA $163(134,187.75)$ vs. CS $159(135,183) ; P$

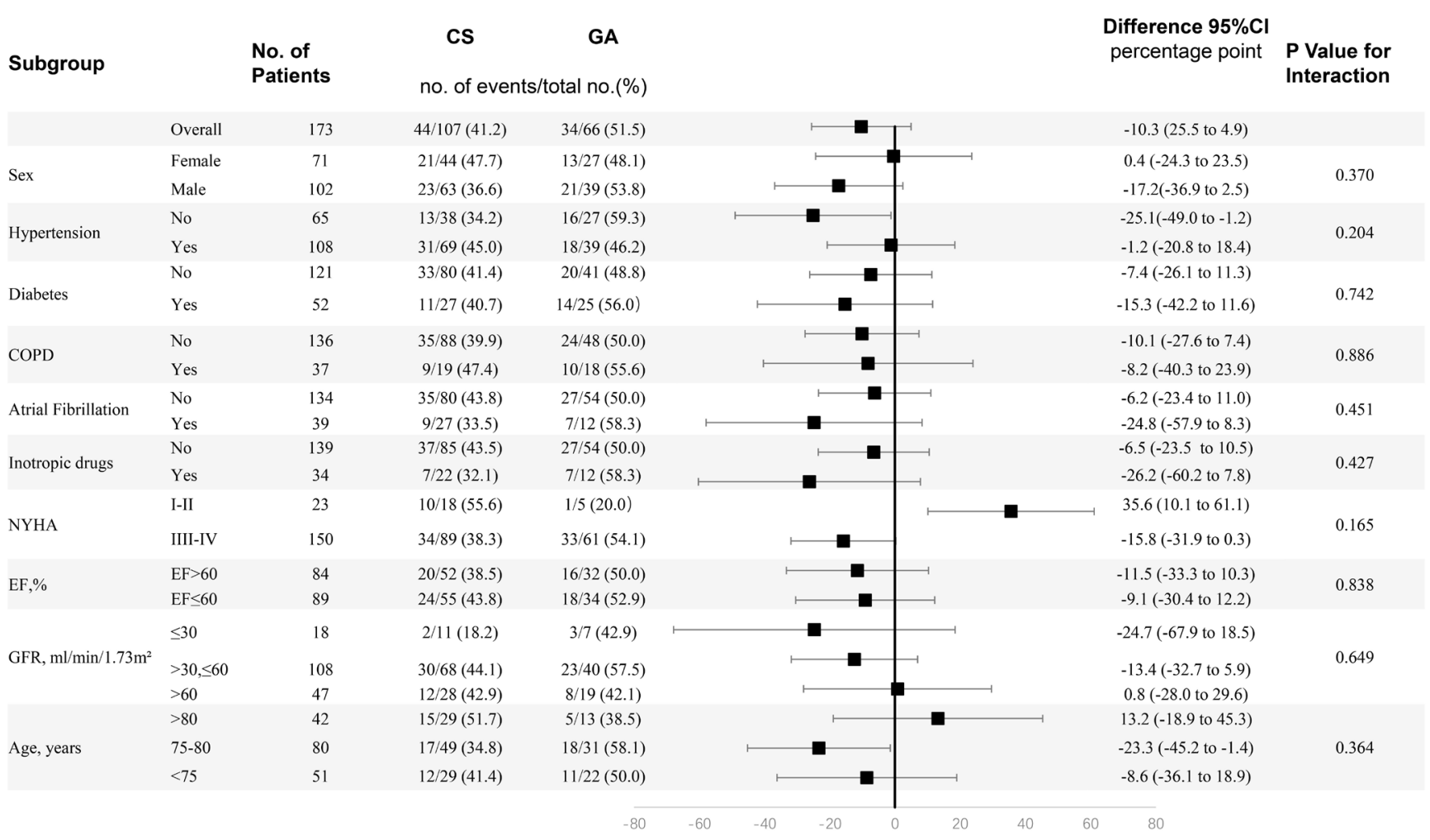

Figure 1. Subgroup analyses of the composite endpoint. Note: All percentages are Kaplan-Meier estimates. Cl indicates confidence interval; COPD, chronic obstructive pulmonary disease; CS, conscious sedation; EF, ejection fraction; GA, general anesthesia; GFR, glomerular filtration rate; NYHA, New York Heart Association. 


Diabetes
Age
ACEI or ARB drugs
NYHA $>\|$
GA
Peripheral vascular disease
GFR $>60 \mathrm{~m} /$ in $/ 1.73 \mathrm{~m}^{2}$
Anesthetic duration
Reintervention
Male
Transaortic approach

b atrial fibrillation

Age
Diabetes
NYHA $>\| 1$
Reintervention
Peripheral vascular disease
GA
Transaortic approach
PCl
COPD

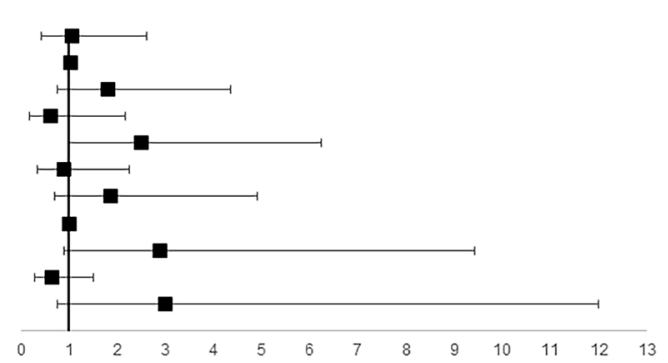

$\begin{array}{ccc}\text { OR } & \mathbf{9 5 \%} \mathrm{Cl} & \begin{array}{c}\text { P Value } \\ 1.064\end{array} \\ 1.048 & 0.931-2.625 & 0.893 \\ 1.819 & 0.757-4.369 & 0.242 \\ 0.634 & 0.185-2.165 & 0.181 \\ 2.517 & 1.013-6.250 & 0.467 \\ 0.889 & 0.352-2.244 & 0.803 \\ 1.865 & 0.708-4.913 & 0.207 \\ 1.005 & 0.996-1.013 & 0.282 \\ 2.897 & 0.891-9.419 & 0.077 \\ 0.640 & 0.274-1.500 & 0.305 \\ 3.016 & 0.757-12.021 & 0.118\end{array}$

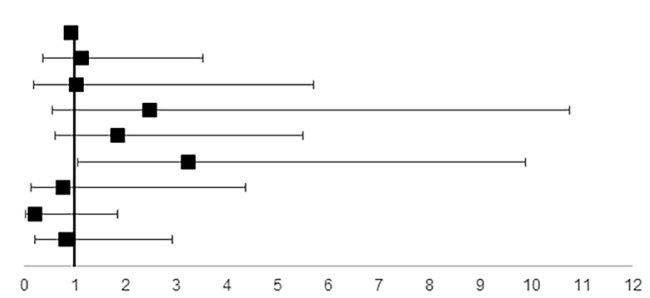

$\begin{array}{ccc}\text { OR } & 95 \% \mathrm{Cl} & \text { P Value } \\ 0.941 & 0.865-1.024 & 0.161 \\ 1.149 & 0.373-3.543 & 0.809 \\ 1.046 & 0.191-5.711 & 0.959 \\ 2.486 & 0.573-10.781 & 0.224 \\ 1.854 & 0.623-5.511 & 0.267 \\ 3.237 & 1.059-9.894 & 0.039 \\ 0.777 & 0.138-4.376 & 0.775 \\ 0.222 & 0.027-1.848 & 0.164 \\ 0.823 & 0.231-2.930 & 0.763\end{array}$

Figure 3. Predictors of AF and AKI. ACEl indicates angiotensin converting enzyme inhibitor; AF, atrial fibrillation; AKI, acute kidney injure; ARB, angiotensin receptor blocker; $\mathrm{Cl}$, confidence interval; COPD, chronic obstructive pulmonary disease; GA, general anesthesia; GFR, glomerular filtration rate; NYHA, New York Heart Association; OR, odds ratio; $\mathrm{PCI}$, percutaneous coronary intervention.

$=.645]$, or postoperative length of stay [GA $9(7,13)$ vs. CS $8(7,13) ; P=.081]$. Additionally, TA-TAVR had the shortest operative duration compared with TF-TAVR (adjusted $P=$ .025 ) and TAO-TAVR (adjusted $P=.001$ ), whereas postoperative length of stay in the TF-TAVR subgroup remained briefer than TA-TAVR (adjusted $P=.032$ ) and TAO-TAVR (adjusted $P<.001$ ) (Tables 2, 3, and S9).

The composite endpoint occurred in 44 patients $(41.2 \%)$ in the CS group compared with 34 patients $(51.5 \%)$ in the GA group. Subgroup analyses of the primary endpoint displayed no heterogeneity of treatment effect in any of the subgroups (Figure 1). Overall, there was no significant difference between GA and CS in all-cause mortality, stroke, PPI, thrombosis, or surgical readmission. At 30 days, GA cohort had a higher rate of major vascular complication (GA $7.7 \%$ vs. CS $0.9 \% ; P=.021$ ), the difference mainly from TAO-TAVR $(60 \%)$. The change of GFR is shown in Figure 2. It is worth noting that the GA group had a significantly higher rate of AKI $(28.8 \%$ vs. $14.0 \%, P=.018)$ and new-onset $\mathrm{AF}(15.2 \%$ vs. $5.5 \%$ at 1 year, $P=.036$ ) (Tables 2, 3, and S9).

\section{Logistic Regression}

Adjusted multilevel logistic regression confirmed GA to be a significant predictor of new-onset $\mathrm{AF}$ [odds ratio (OR) 3.237, $95 \%$ confidence interval (CI) 1.059 to $9.894 ; P=.039]$. Factors including age $(P=.161)$, diabetes $(P=.809)$, NYHA class $>$ II $(P$ $=.959)$, reintervention $(P=.224)$, peripheral vascular disease $(P$ $=.267)$, TAO approach $(P=.775)$, PCI $(P=.164)$, and COPD $(P=.763)$ displayed no statistical impact on postoperative newonset AF. In another adjusted regression model for predicting a paravalvular leakage between GA and CS groups

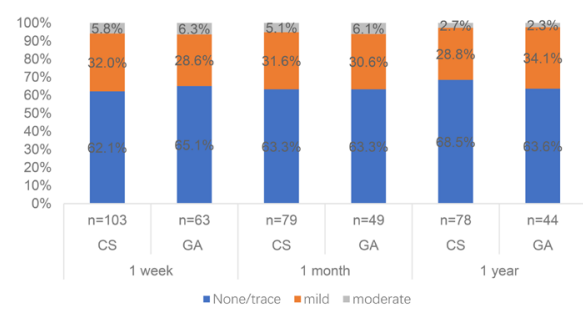

b $\geq$ mild paravalvular leakage among different approaches over time in GA subgroup

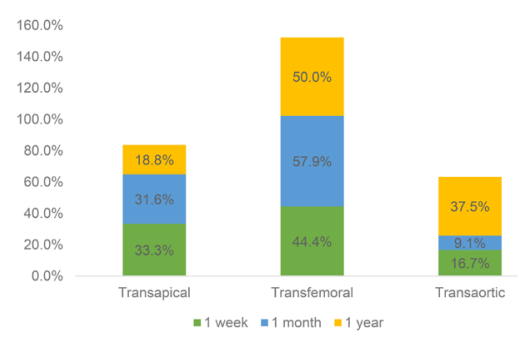

Figure 4. PVL based on 2 anesthetic strategies (a) and different approaches (b). CS indicates conscious sedation; GA, general anesthesia; $\mathrm{PVI}$, paravalvular leakage.

AKI, GA was proved an independent predictor (OR 2.517, $95 \%$ CI 1.013 to $6.250, P=.047)$. No significant difference was observed in diabetes $(P=.893)$, age $(P=.242)$, enzyme inhibitor (ACEI)/angiotensin receptor blocker (ARB) drugs within 48 hours $(P=.181)$, NYHA $>$ II $(P=.467)$, peripheral vascular 
Table 2. Postoperative Outcomes Based on the Entire Sample*

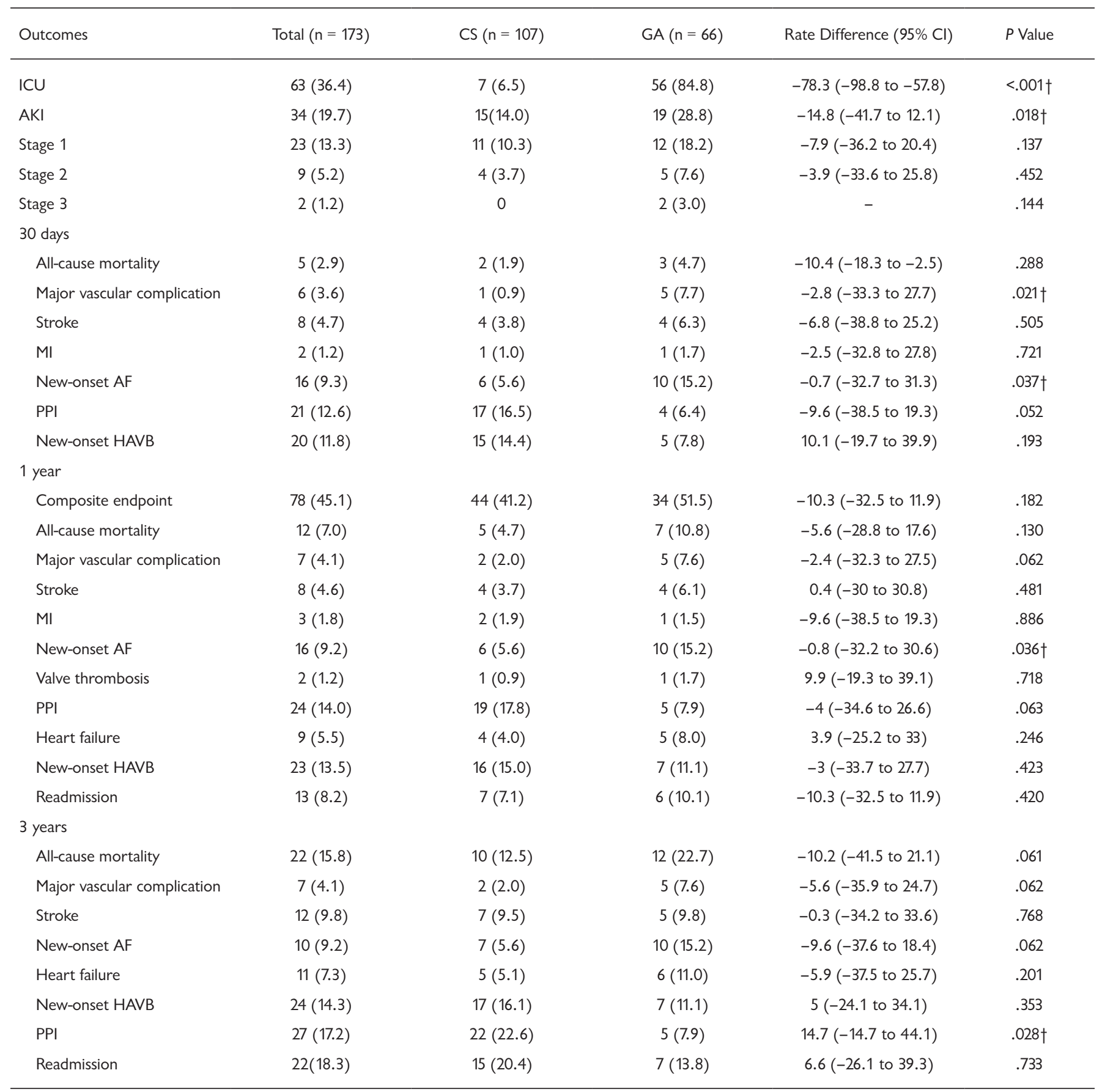

*Values are $\mathrm{n}(\%)$ unless noted otherwise. Values at 30 days, 1 year, and 3 years were calculated by Kaplan-Meier curves.

†Significant.

$\mathrm{AF}$ indicates atrial fibrillation; $\mathrm{AKI}$, acute kidney injury; $\mathrm{Cl}$, confidence interval; $\mathrm{CS}$, conscious sedation; GA, general anesthesia; HAVB, high atrioventricular block; ICU, intensive care unit; MI, myocardial infarction; PPI, permanent pacemaker implantation. 
Table 3. Postoperative Outcomes of 3 Different Approaches under GA*

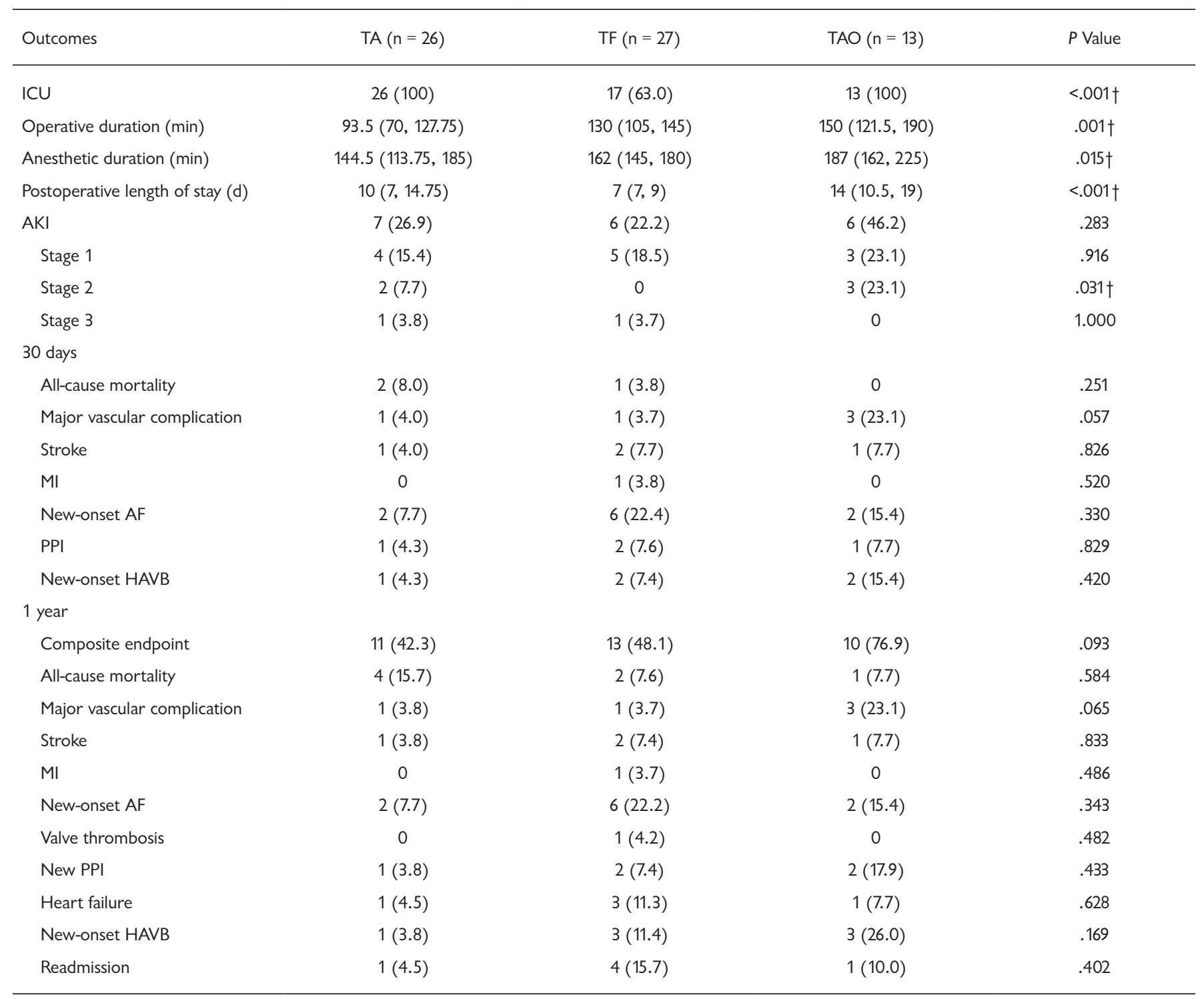

*Values are $\mathrm{n}(\%)$ or median (interquartile range). Values at 30 days and 1 year were calculated by Kaplan-Meier curves.

†Significant.

AF indicates atrial fibrillation; AKI, acute kidney injury; $\mathrm{Cl}$, confidence interval; $\mathrm{CS}$, conscious sedation; GA, general anesthesia; HAVB, high atrioventricular block; ICU, intensive care unit; MI, myocardial infarction; PPI, permanent pacemaker implantation; TA, transapical; TAO, transaortic; TF, transfemoral.

\section{Supplemental Material}

\section{Variables Enrolled in Multivariate Regression Models}

AF: age, COPD, GA, reintervention, NYHA class $>I I$, peripheral vascular disease, diabetes, TAO approach

AKI: GA, age, diabetes, NYHA class >II, GFR $>60 \mathrm{~mL} / \mathrm{min} / 1.73 \mathrm{~m} 2$, anesthetic duration, peripheral vascular disease, reintervention, ACEI/ARB drugs within $48 \mathrm{~h}$, TAO approach, male

\section{Selected Method}

First, crucial preoperative variables were brought into univariate logistic regression analysis to determine the association with the dependent variable. Then variables that were significant $(\mathrm{P}<.1)$ on univariate analysis or clinically relevant were considered to construct the multivariate logistic regression. 
disease $(P=.803)$, glomerular filtration rate $(\mathrm{GFR})>60 \mathrm{~mL} /$ $\min / 1.73 \mathrm{~m} 2(P=.207)$, anesthetic duration $(P=.282)$, reintervention $(P=.077)$, male $(P=.305)$, or TAO approach $(P=.118)$ (Figure 3$)$. We also set an adjusted model exclusively based on TF-TAVR to avoid the bias that comes with approach. Results revealed the effect of GA on AF (OR 5.193, 95\% CI 1.288 to $20.943, P=.021$ ) and AKI (OR 4.596, 95\% CI 1.167 to $18.096, P=.029)$ still remained in the TF TAVR despite that the sample was limited (Table S10 and S11).

\section{Paravalvular Leakage}

Available data exhibited that there was no significant difference in $\geq$ mild PVL between GA and CS at 1 week (GA $34.9 \%$ vs. CS $37.9 \% ; P=.703$ ), 1 month (GA $36.7 \%$ vs. CS $36.7 \% ; P=.998$ ), and 1 year (GA $36.4 \%$ vs. CS $31.4 \% ; P=$ .589). In regard to TAVR under GA, $\geq$ mild PVL in the TF subgroup (44.4\% to $50.0 \%$ ) was slightly higher than TAO (16.7\% to $37.5 \%)$ and TA (18.8\% to $33.3 \%)$ subgroups, despite that there was no statistical difference among the 3 subgroups (Figure 4, Tables S12-S14).

\section{DISCUSSION}

AKI occurred in $20.7 \%$ to $41.7 \%$ of patients after TAVR and was associated with increased mortality [Attard 2018; Kumar 2019; Nuis 2012; Saia 2013]. Underlying risk factors of AKI involved chronic kidney disease (CKD), higher median EuroSCORE-II, peripheral artery disease, prior coronary artery bypass grafting, number of blood transfusions $\leq 24$ hours [Aalaei-Andabili 2016; Attard 2018; Nuis 2012]. The current study presented GA as an additional predictor of AKI. This was explainable, in that theoretically patients under GA are more likely to experience AKI because of the management of multiple anesthetics and prolonged fluoroscopy times. In this study, TAO-TAVR was associated with statistically nonsignificant increases of AKI (TAO 46.2\%, TA 26.9\%, TF 22.2\%; $P=.295$ ). This was not unique: according to the report of Cocchieri et al. [2019], the occurrence of stage 2/3 AKI in TAO TAVR accounted for a high rate of $14.7 \%$. Studies have shown non-TF access to result in higher rates of AKI than TF access, largely because of the more invasive approach resulting in a greater need for blood transfusions, which appeared to have a direct harmful effect on the kidneys [Aalaei-Andabili 2016; Cocchieri 2019; Nuis 2012]. It should be underlined that anesthesiologists and operators are more prudent in dealing with patients under GA or via a TAO approach in the effort to minimize the known risk of AKI.

AF was detected in a high proportion following aortic valve replacement [Helgadottir 2012]. AF was connected with prolonged length of hospital stay, increased risk of stroke and mortality [Ahlsson 2010; Aranki 1996; Villareal 2004]. It has been reported that age, female, hypertension, $\mathrm{COPD}$, and $\mathrm{AF}$ history are relevant to postoperative $\mathrm{AF}$ [Ahlsson 2010; Mathew 2004]. In this research, rather than diabetes, peripheral vascular disease, or NYHA, GA was the
Table S4. Test of Multicollinearity

\begin{tabular}{lll}
\hline Factor & Tolerance & VIF \\
\hline Acute kidney injury & & \\
Age & 0.841 & 1.189 \\
Male & 0.931 & 1.074 \\
GA & 0.815 & 1.228 \\
Diabetes & 0.924 & 1.082 \\
NYHA class >II & 0.919 & 1.088 \\
Peripheral vascular disease & 0.909 & 1.100 \\
Reintervention & 0.905 & 1.105 \\
ACEI/ARB drugs within $48 \mathrm{~h}$ & 0.975 & 1.026 \\
GFR >60 mL/min/1.73 m2 & 0.833 & 1.200 \\
Anesthetic duration & 0.899 & 1.113 \\
TAO approach & 0.813 & 1.230 \\
AF & & \\
Age & 0.946 & 1.057 \\
Diabetes & 0.896 & 1.116 \\
GA & 0.801 & 1.249 \\
NYHA class >II & 0.923 & 1.083 \\
Peripheral vascular disease & 0.916 & 1.091 \\
Reintervention & 0.936 & 1.068 \\
COPD & 0.945 & 1.058 \\
PCI & 0.930 & 1.075 \\
TAO approach & 0.853 & 1.173 \\
\hline
\end{tabular}

ACEl indicates angiotensin converting enzyme inhibitors; $A F$, atrial fibrillation $\mathrm{ARB}$, angiotensin receptor blocker; COPD, chronic obstructive pulmonary disease; GA, general anesthesia; GFR, glomerular filtration rate; NYHA, New York Heart Association; $\mathrm{PCl}$, percutaneous coronary intervention.

exclusively independent predictor of AF. The mechanism of AF was intricate, possible explanations involving rapidly excessive activation of sympathetic nervous systems following deep suppression during operation, circulatory fluctuation, anaphylaxis resulting from general anesthetics. Some findings suggested that beta-blockers, ACEI, or nonsteroidal antiinflammatory medications might offer protection [Mathew 2004]. Additional studies will be needed to better identify and prevent the occurrence of AF.

The overwhelming advantage to GA is the ability to control ventilation and enable real-time TEE guidance. It has been said to be associated with significantly less PVL compared with CS [Oguri 2014; Zaouter 2018]. Such a difference is probably related to the insertion of a TEE probe during GA that guides the correct valve deployment and detects PVL requiring postimplantation dilation [Bagur 2011; Zaouter 2018]. Intraprocedural TEE has been advised as a helpful adjunct for valve positioning, assessment of 
Table S5. Univariate Analysis in AKI

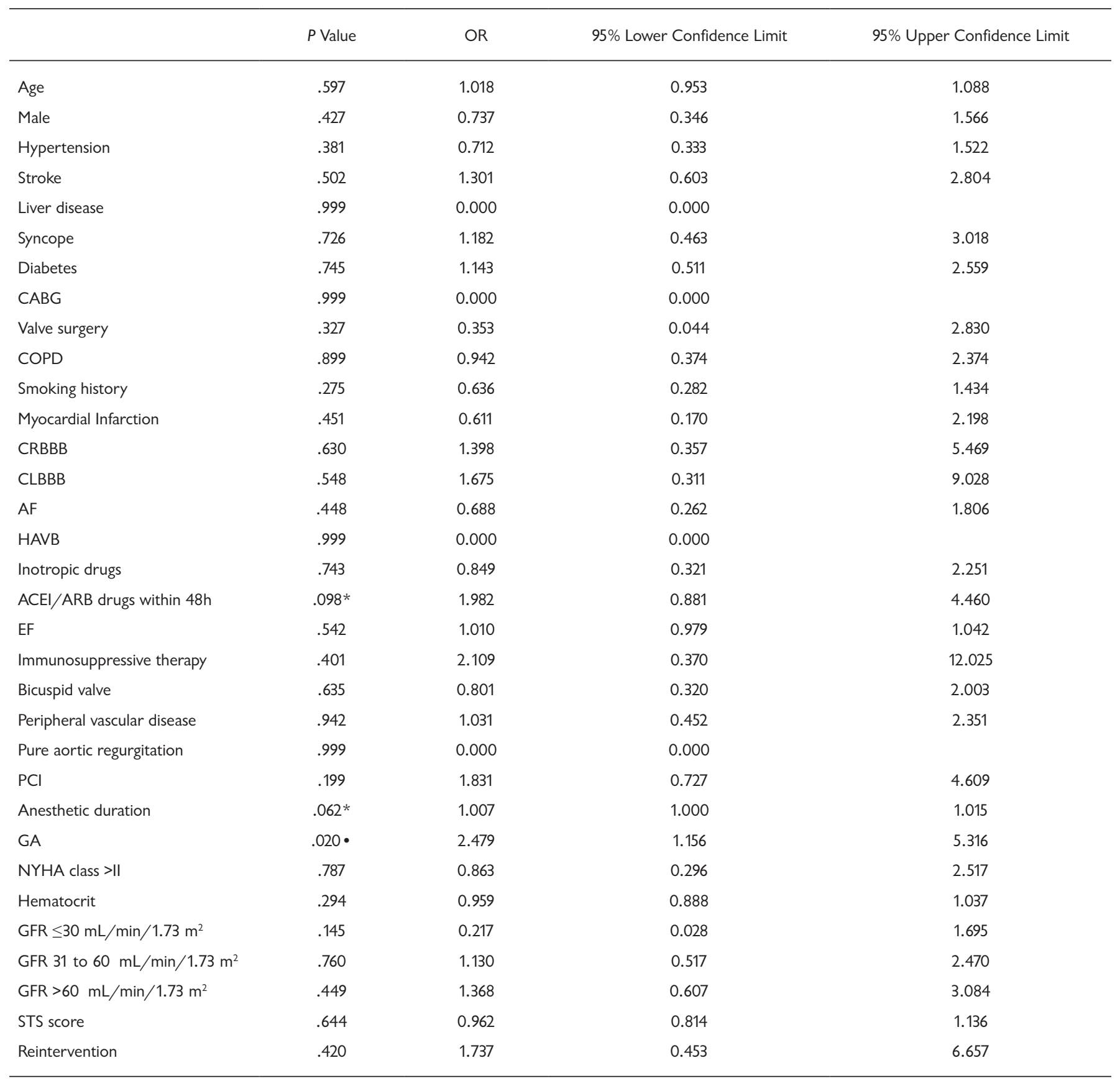

*Significant.

ACEl indicates angiotensin converting enzyme inhibitors; $A F$, atrial fibrillation; $A R B$, angiotensin receptor blocker; $C A B G$, coronary-artery bypass surgery; CLBBB, complete left bundle branch block; COPD, chronic obstructive pulmonary disease; CRBBB, complete right bundle branch block; $E F$, ejection fraction GA, general anesthesia; GFR, glomerular filtration rate; HAVB, high-grade atrioventricular block; NYHA, New York Heart Association; OR, odds ratio; PCl, percutaneous coronary intervention; STS, Society of Thoracic Surgeons. 
Table S6. Univariate Analysis in AF

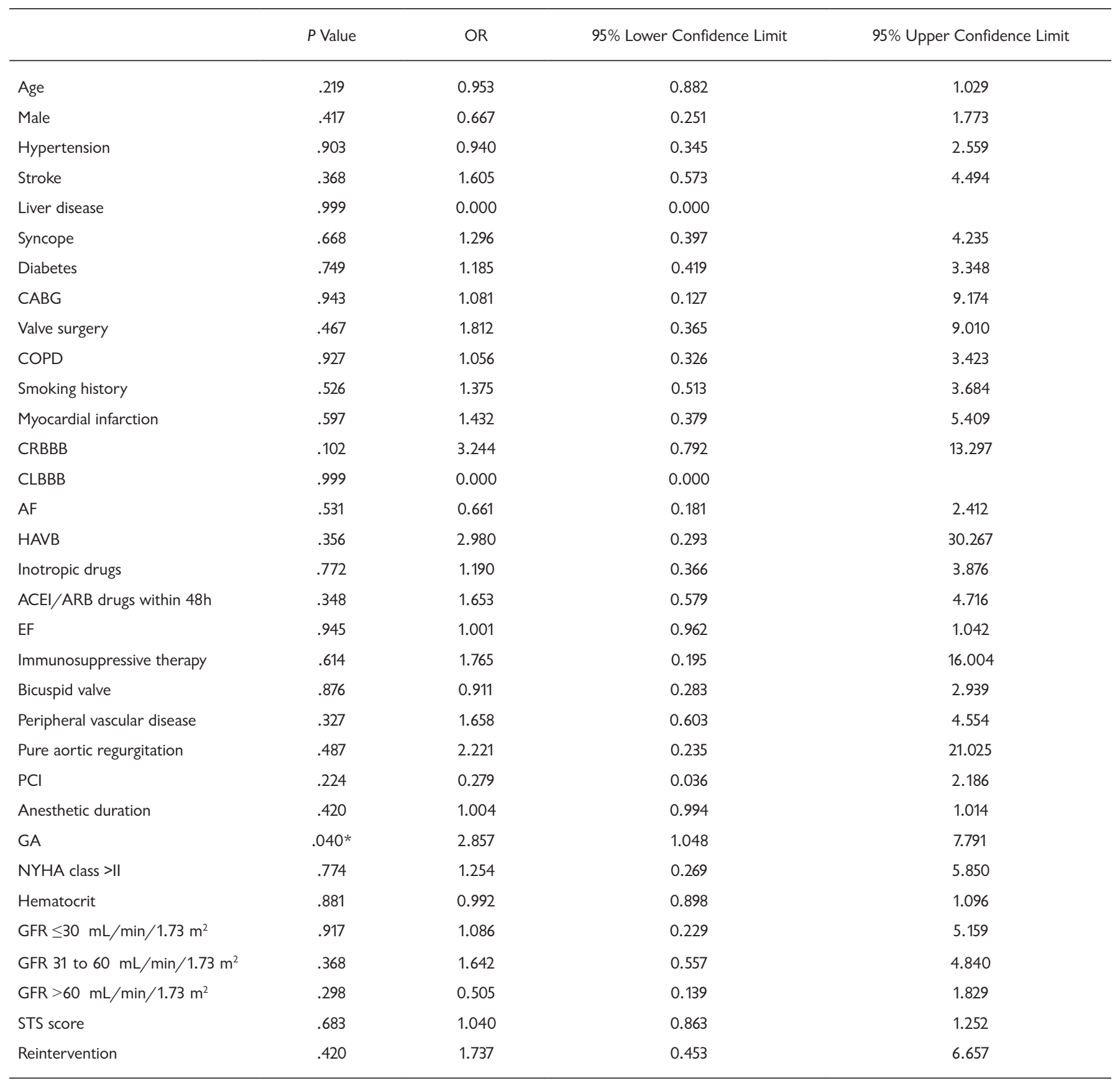

*Significant.

$A C E l$ indicates angiotensin converting enzyme inhibitors; $A F$, atrial fibrillation; $A R B$, angiotensin receptor blocker; $C A B G$, coronary-artery bypass surgery; CLBBB, complete left bundle branch block; COPD, chronic obstructive pulmonary disease; CRBBB, complete right bundle branch block; $E F$, ejection fraction GA, general anesthesia; GFR, glomerular filtration rate; HAVB, high-grade atrioventricular block; NYHA, New York Heart Association; OR, odds ratio; PCl, percutaneous coronary intervention; STS, Society of Thoracic Surgeons. 
Table S7. Baseline Characteristics of 3 Different Approaches*

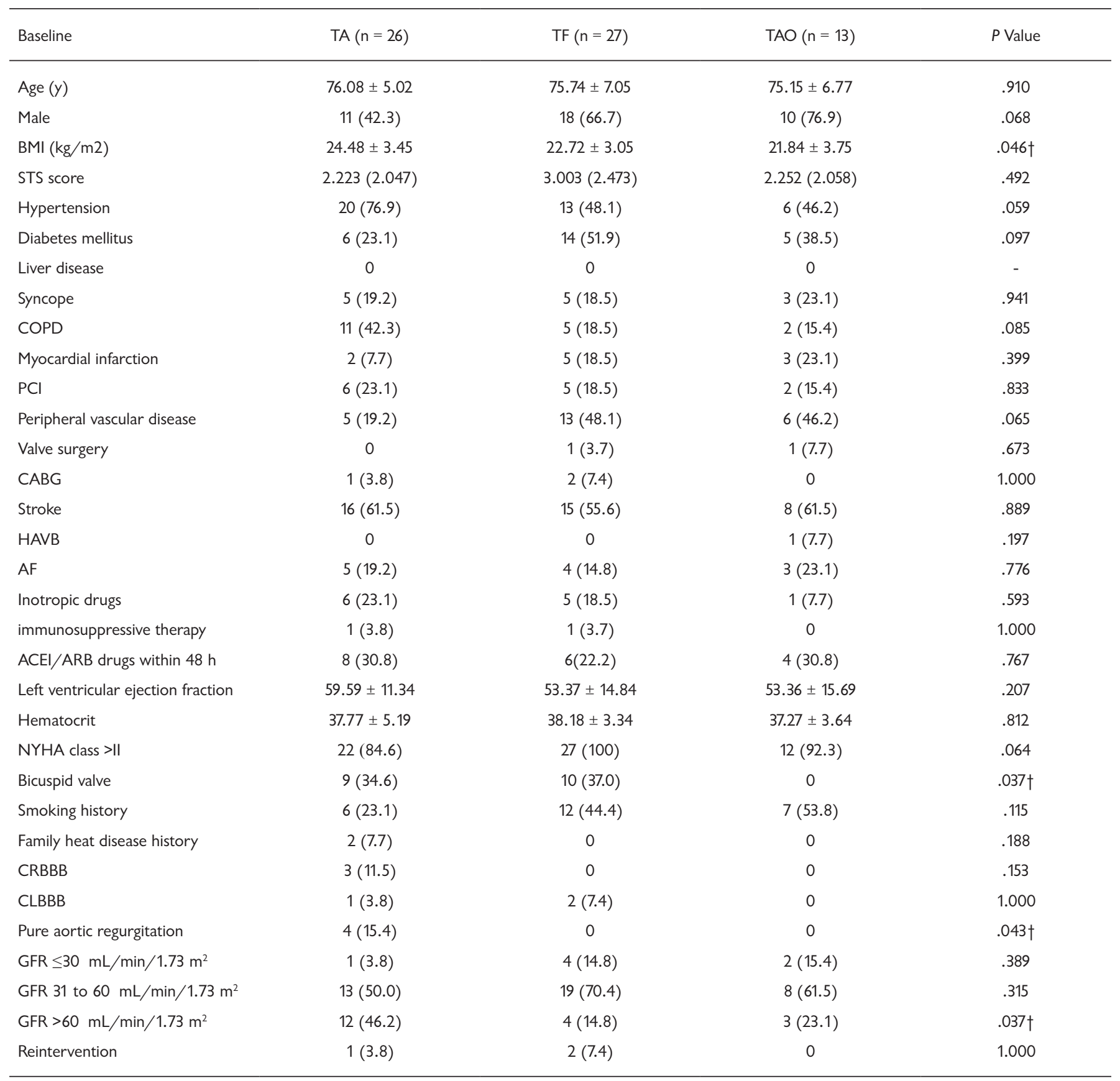

*Values are mean $\pm S D$, median (interquartile range), or $\mathrm{n}(\%)$.

†Significant.

ACEl indicates angiotensin converting enzyme inhibitors; AF, atrial fibrillation; ARB, angiotensin receptor blocker; BMI, body mass index; CABG, coronaryartery bypass surgery; CLBBB, complete left bundle branch block; COPD, chronic obstructive pulmonary disease; CRBBB, complete right bundle branch block; GA, general anesthesia; GFR, glomerular filtration rate; HAVB, high-grade atrioventricular block; NYHA, New York Heart Association; PCI, previous percutaneous intervention; STS, Society of Thoracic Surgeons; TA, transapical; TAO, transaortic; TF, transfemoral. 
Table S8. Baseline Characteristics of TF-TAVR*

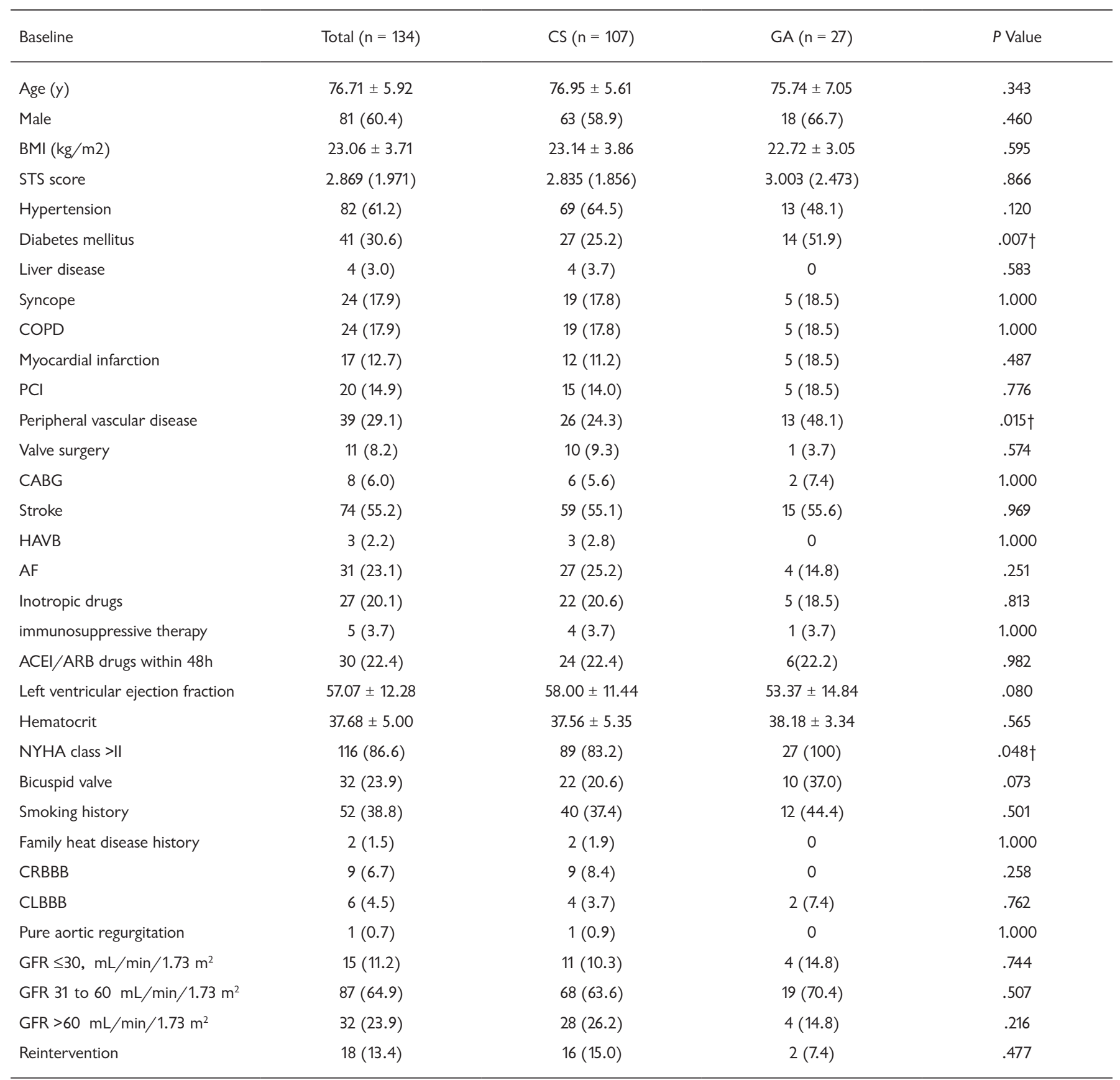

*Values are mean $\pm S D$, median (interquartile range), or $\mathrm{n}(\%)$.

†Significant.

ACEl indicates angiotensin converting enzyme inhibitors; AF, atrial fibrillation; ARB, angiotensin receptor blocker; BMI, body mass index; CABG, coronaryartery bypass surgery; CLBBB, complete left bundle branch block; COPD, chronic obstructive pulmonary disease; CRBBB, complete right bundle branch block; CS, conscious sedation; GA, general anesthesia; GFR, glomerular filtration rate; HAVB, high-grade atrioventricular block; NYHA, New York Heart Association; $\mathrm{PCl}$, percutaneous coronary intervention; STS, Society of Thoracic Surgeons; TAVR, transcatheter aortic valve replacement; TF, transfemoral. 
Table S9. Postoperative Outcomes of TF Transcatheter Aortic Valve Replacement*

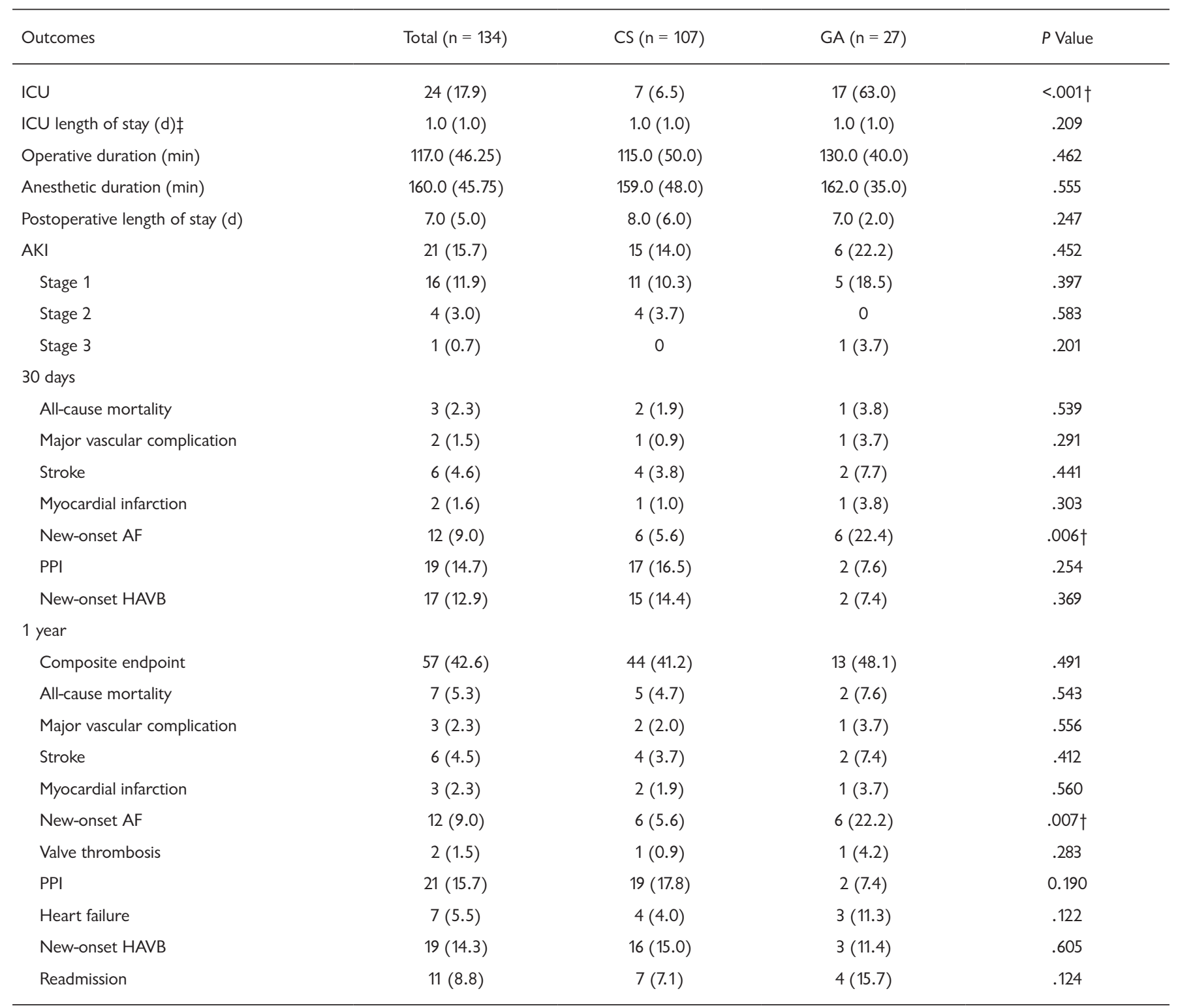

*Values are $\mathrm{n}(\%)$.

†Significant.

$\ddagger$ Calculated among patients admitted to ICU,not including those back to ward immediately after procedure.

$\mathrm{AF}$ indicates atrial fibrillation; AKI, acute kidney injury; CS, conscious sedation; GA, general anesthesia; ICU, intensive care unit; HAVB, high-grade atrioventricular block; PPI, permanent pacemaker implantation; TAVR, transcatheter aortic valve replacement; TF, transfemoral.

Complications are defined according to the Valve Academic Research Consortium-2 consensus document; Values at 30 days and 1 year were calculated by Kaplan-Meier curves. 
Table S10. Predictors of AF in TF-TAVR

\begin{tabular}{lcccc}
\hline & $P$ Value & OR & 95\% Lower Confidence Limit & 95\% Upper Confidence Limit \\
\hline Diabetes & .783 & 1.207 & 0.317 & 4.600 \\
Age & .429 & 0.958 & 0.863 & 1.065 \\
NYHA class >II & .205 & 0.287 & 0.041 & 1.978 \\
GA & $.021^{*}$ & 5.193 & 1.288 & 20.943 \\
Peripheral vascular disease & .401 & 1.811 & 0.453 & 7.241 \\
Reintervention & .139 & 3.257 & 0.683 & 15.533 \\
PCl & .341 & 0.336 & 0.036 & 3.167 \\
COPD & .757 & 0.771 & 0.148 & 4.014 \\
\hline
\end{tabular}

*Significant.

AF indicates atrial fibrillation; COPD, chronic obstructive pulmonary disease; GA, general anesthesia; PCl, percutaneous coronary intervention; OR, odds ratio; TAVR, transcatheter aortic valve replacement; TF, transfemoral.

Table S11. Predictors of AKI in TF-TAVR

\begin{tabular}{lllll}
\hline & $P$ Value & OR & 95\% Lower Confidence Limit & 95\% Upper Confidence Limit \\
\hline Diabetes & .177 & 0.405 & 0.109 & 1.504 \\
Age & .623 & 1.026 & 0.925 & 1.138 \\
ACEl or ARB drugs & .110 & 2.588 & 0.806 & 8.308 \\
NYHA class $>I I$ & .203 & 0.359 & 0.074 & 1.739 \\
GA & $.029^{*}$ & 4.596 & 1.167 & 18.096 \\
Peripheral vascular disease & .476 & 1.583 & 0.447 & 5.607 \\
GFR $>60 \mathrm{~mL} / \mathrm{min} / 1.73 \mathrm{~m}^{2}$ & .095 & 3.297 & 0.814 & 13.357 \\
Anesthetic duration & .789 & 1.002 & 0.989 & 1.015 \\
Reintervention & .006 & 6.891 & 1.729 & 0.053 \\
Male & .005 & 0.178 & 0.053 & \\
\hline
\end{tabular}

*Significant.

ACEl indicates angiotensin converting enzyme inhibitors; AKI, acute kidney injury; ARB, angiotensin receptor blocker; GA, general anesthesia; GFR, glomerular filtration rate; NYHA, New York Heart Association; OR, odds ratio; TAVR, transcatheter aortic valve replacement TF, transfemoral.

results, and detection of complications [Durand 2012]. In our center, TEE was exclusively applied in TAVR under GA, whereas in the CS group, TTE and fluoroscopy are the main tools for intraprocedural imaging. However, results revealed there was no significant difference between CS and GA in $\geq$ mild PVL. Similarly, Zaouter's report concluded that performing TAVR under GA with TEE guidance is not associated with a lower incidence of moderate and severe PVL [Zaouter 2018]. Furthermore, GA is not requisite for TEE. On the one hand, successful TEE-guided TAVR performed under CS has been reported elsewhere [Ben-Dor 2012; Kiramijyan 2016]. On the other hand, new imaging alternatives are emerging. Intracardiac echocardiography (ICE) allowed for the evaluation of perioperative hemodynamics, measurement of the aortic valve complex, and assessment of major complications during the procedure without interference from the operator or fluoroscopes [Yagasaki 2018]. It has been reported that ICE, which is compatible with sedation and local anesthesia, can be considered an alternative to TEE for intraprocedural guidance during TAVR and match the required workflow during TAVR better than TEE [Bartel 2011; Kadakia 2015]. Imaging guidance with the use of ICE will be an important step in moving toward performing TAVR under CS.

\section{Limitations}

This research has limitations. First, it was a single-center, retrospective, and nonrandomized controlled study. Second, given the small sample from the population in our center, additional studies will be needed to better characterize the 
Table S12. PVL Based on the Entire Sample*

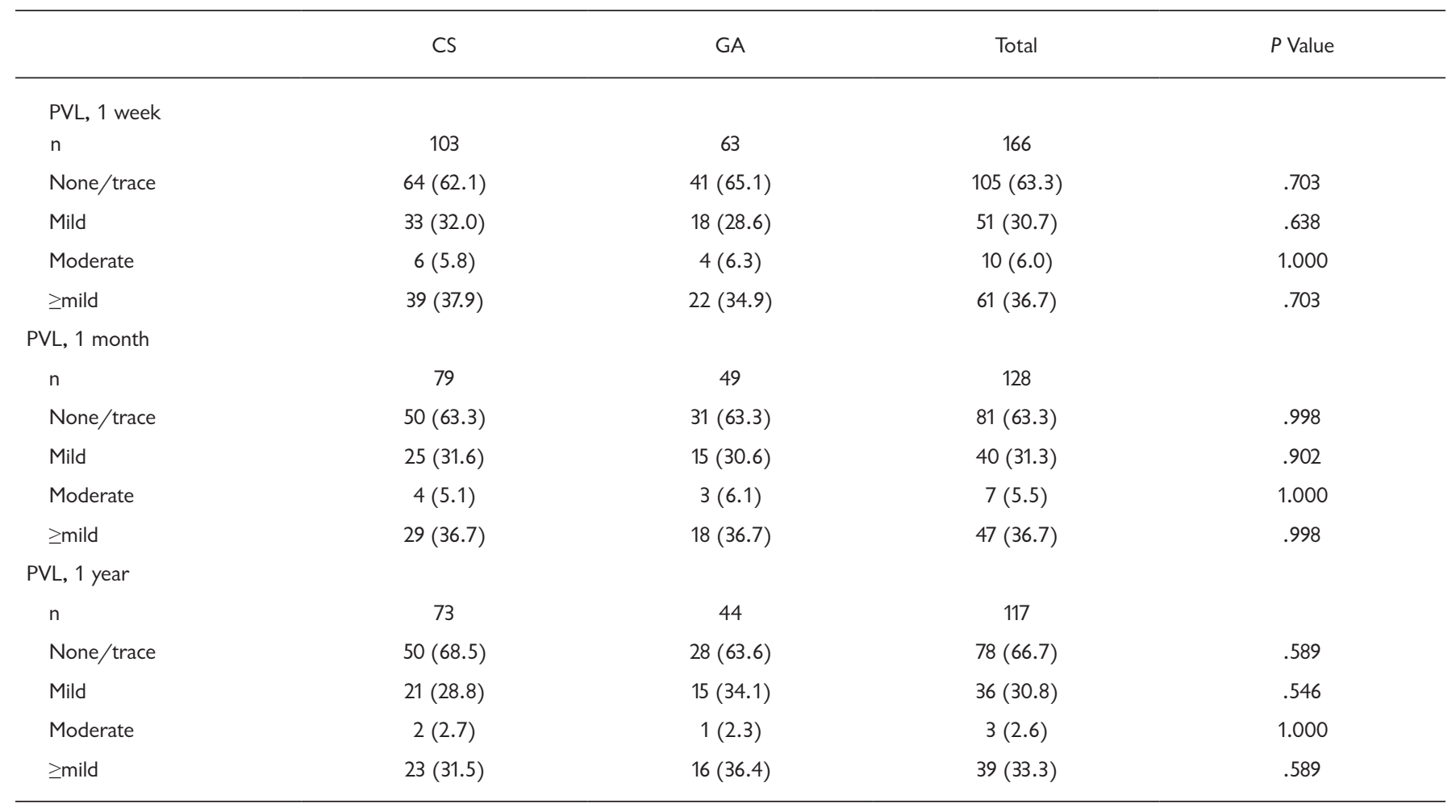

*Values are $\mathrm{n}(\%)$.

CS indicates conscious sedation; GA, general anesthesia; PVL, paravalvular leakage.

risk factors for and predictors of $\mathrm{AF}$ and $\mathrm{AKI}$. Last, the identification of PVL merely relied on echocardiographic findings in Fuwai Hospital. Because some patients went back to local hospitals for further examinations, we were not able to obtain comprehensive data.

\section{Conclusion}

GA was an independent predictor of postoperative AKI and new-onset AF. This study may provide new evidence to challenge the universal of general anesthesia and supports the hypothesis that TAVR with conscious sedation is associated with superior clinical outcomes in comparison with TAVR with GA.

\section{ACKNOWLEDGEMENTS}

The authors thank Can Cai, Key Laboratory of Cardiovascular Epidemiology \& Department of Epidemiology, for her statistic support and help. Wish all the best in her work.

\section{FUNDING}

This work was supported by grants from the National Natural Science Foundation of China (Grant No. 8167010988) and College Student-funding in Peking Union Medical College (No.2019-1002-77). 
Table S13. PVL of 3 Different Approaches*

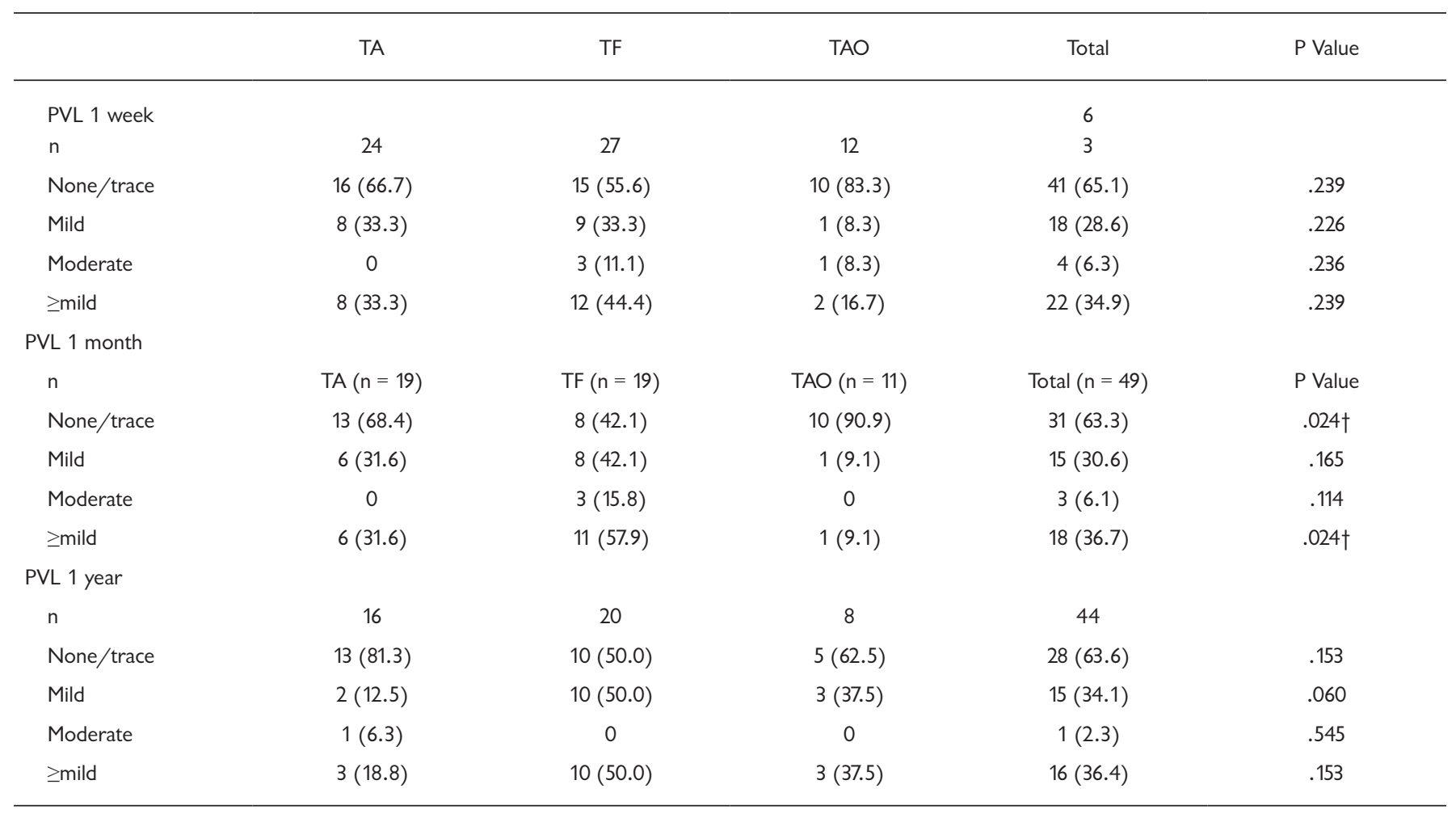

*Values are $n(\%)$.

† significant values. PVL indicates paravalvular leakage; TA, transapical; TAO, transaortic; TF, transfemoral. 
The Heart Surgery Forum 2021-3361

Table S14. PVL of TF-TAVR*

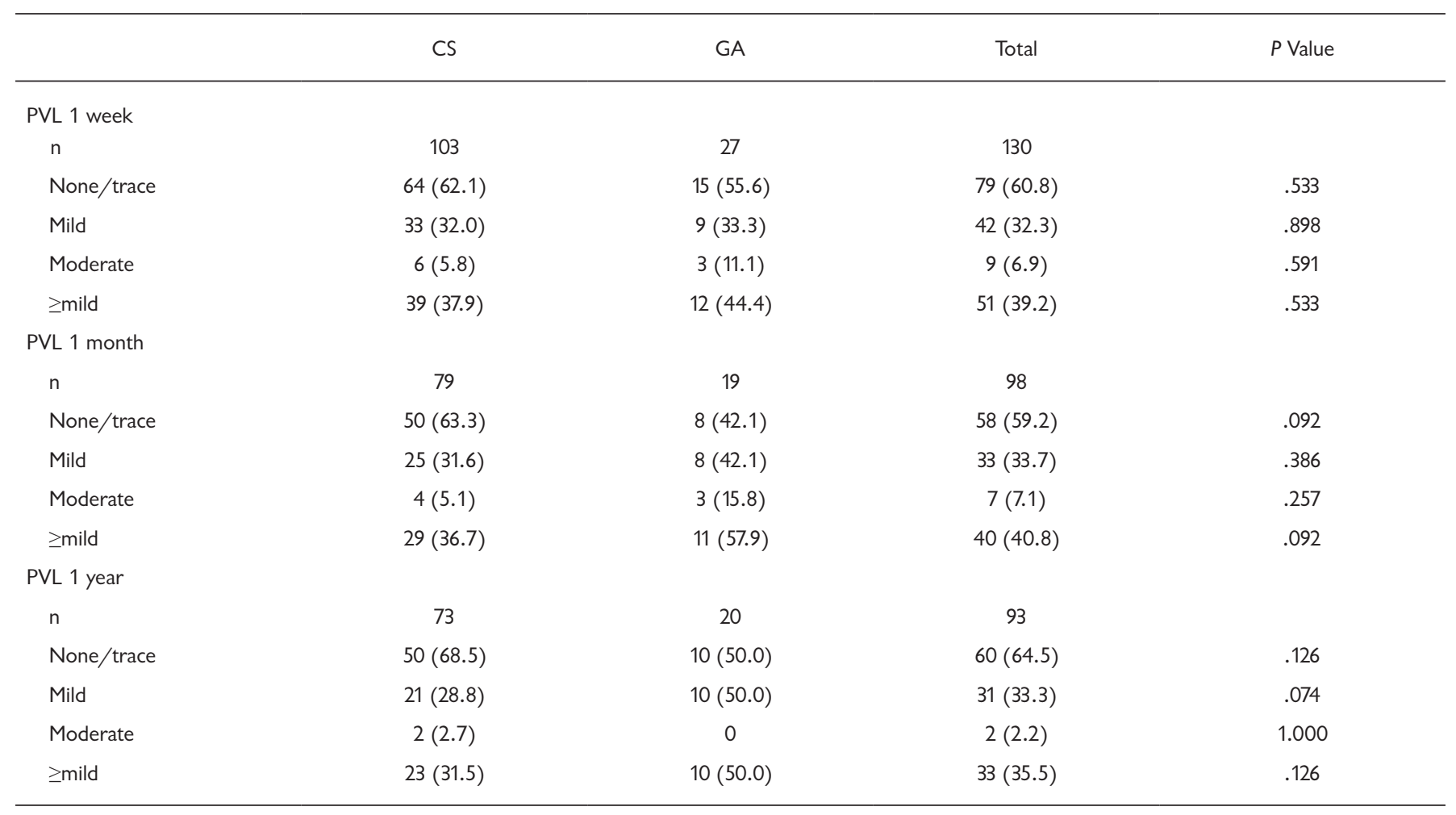

*Values are $\mathrm{n}(\%)$.

CS indicates conscious sedation; GA, general anesthesia; PVI, paravalvular leakage; TAVR, transcatheter aortic valve replacement, TF, transfemoral. 


\section{REFERENCES}

Aalaei-Andabili SH, Pourafshar N, Bavry AA, et al. Acute kidney injury after transcatheter aortic valve replacement. J Card Surg 2016;31:416-422.

Ahlsson A, Fengsrud E, Bodin L, Englund A. Postoperative atrial fibrillation in patients undergoing aortocoronary bypass surgery carries an eightfold risk of future atrial fibrillation and a doubled cardiovascular mortality. Eur J Cardiothorac Surg 2010;37:1353-1359.

Aranki SF, Shaw DP, Adams DH, et al. Predictors of atrial fibrillation after coronary artery surgery. Current trends and impact on hospital resources. Circulation 1996;94:390-397.

Attard S, Buttigieg J, Galea S, et al. The incidence, predictors, and prognosis of acute kidney injury after transcatheter aortic valve implantation. Clin Nephrol 2018;90:373-379.

Attizzani GF, Alkhalil A, Padaliya B, et al. Comparison of outcomes of transfemoral transcatheter aortic valve implantation using a minimally invasive versus conventional strategy. Am J Cardiol 2015;116:1731-1736.

Attizzani GF, Patel SM, Dangas GD, et al. Comparison of local versus general anesthesia following transfemoral transcatheter self-expanding aortic valve implantation (from the transcatheter valve therapeutics registry). Am J Cardiol 2019;123:419-425.

Bagur R, Rodes-Cabau J, Doyle D, et al. Usefulness of tee as the primary imaging technique to guide transcatheter transapical aortic valve implantation. JACC Cardiovasc Imaging 2011;4:115-124.

Bainbridge D, Martin J, Arango $M$ et al. Perioperative and anaestheticrelated mortality in developed and developing countries: A systematic review and meta-analysis. Lancet 2012;380:1075-1081.

Bartel T, Bonaros N, Muller L, et al. Intracardiac echocardiography: A new guiding tool for transcatheter aortic valve replacement. J Am Soc Echocardiogr 2011;24:966-975.

Ben-Dor I, Looser PM, Maluenda G, et al. Transcatheter aortic valve replacement under monitored anesthesia care versus general anesthesia with intubation. Cardiovasc Revasc Med 2012;13:207-210.

Butala NM, Chung M, Secemsky EA, et al. Conscious sedation versus general anesthesia for transcatheter aortic valve replacement: Variation in practice and outcomes. JACC Cardiovasc Interv 2020;13:1277-1287.

Cocchieri R, Petzina R, Romano M, et al. Outcomes after transaortic transcatheter aortic valve implantation: Long-term findings from the European ROUTE†. Eur J Cardiothorac Surg 2019;55:737-743.

Durand E, Borz B, Godin M et al. Transfemoral aortic valve replacement with the Edwards Sapien and Edwards Sapien XT prosthesis using exclusively local anesthesia and fluoroscopic guidance: Feasibility and 30-day outcomes. JACC Cardiovasc Interv 2012;5:461-467.

Helgadottir S, Sigurdsson MI, Ingvarsdottir IL, et al. Atrial fibrillation following cardiac surgery: Risk analysis and long-term survival. J Cardiothorac Surg 2012;7:87.

Hellhammer K, Piayda K, Afzal S et al. The latest evolution of the Medtronic CoreValve system in the era of transcatheter aortic valve replacement: Matched comparison of the Evolut PRO and Evolut R. JACC Cardiovasc Interv 2018;11:2314-2322.

Hyman MC, Vemulapalli S, Szeto WY et al. Conscious sedation versus general anesthesia for transcatheter aortic valve replacement: Insights from the National Cardiovascular Data Registry Society of Thoracic Surgeons/American College of Cardiology transcatheter valve therapy registry. Circulation 2017;136:2132-2140.
Kadakia MB, Silvestry FE, Herrmann HC. Intracardiac echocardiography-guided transcatheter aortic valve replacement. Catheter Cardiovasc Interv 2015;85:497-501.

Kappetein AP, Head SJ, Genereux P et al. Updated standardized endpoint definitions for transcatheter aortic valve implantation: The Valve Academic Research Consortium-2 consensus document (varc-2). Eur J Cardiothorac Surg 2012;42:S45-S60.

Kiramijyan S, Ben-Dor I, Koifman E, et al. Comparison of clinical outcomes with the utilization of monitored anesthesia care vs. general anesthesia in patients undergoing transcatheter aortic valve replacement. Cardiovasc Revasc Med 2016;17:384-390.

Konigstein M, Ben-Shoshan J, Zahler D et al. Outcome of patients undergoing TAVR with and without the attendance of an anesthesiologist. Int J Cardiol 2017;241:124-127.

Kumar N, Garg N. Acute kidney injury after aortic valve replacement in a nationally representative cohort in the USA. Nephrol Dial Transplant 2019;34:295-300

Leon MB, Smith CR, Mack M, et al. Transcatheter aortic-valve implantation for aortic stenosis in patients who cannot undergo surgery. N Engl J Med 2010;363:1597-1607.

Leon MB, Smith CR, Mack MJ et al. Transcatheter or surgical aortic-valve replacement in intermediate-risk patients. N Engl J Med 2016;374:1609-1620.

Maier A, Hammerich B, Humburger F, et al. A logistic regression analysis comparing minimalistic approach and intubation anaesthesia in patients undergoing transfemoral transcatheter aortic valve replacement. PLoS One 2020;15:e0227345.

Manoharan G, Van Mieghem NM, Windecker S, et al. 1-year outcomes with the evolut $\mathrm{r}$ self-expanding transcatheter aortic valve: From the international forward study. JACC Cardiovasc Interv 2018;11:2326-2334.

Marcantuono R, Gutsche J, Burke-Julien M, et al. Rationale, development, implementation, and initial results of a fast track protocol for transfemoral transcatheter aortic valve replacement (TAVR). Catheter Cardiovasc Interv 2015;85:648-654.

Mathew JP, Fontes ML, Tudor IC, et al. A multicenter risk index for atrial fibrillation after cardiac surgery. JAMA 2004;291:1720-1729.

Mosleh W, Mather JF, Amer MR, et al. Propensity matched analysis comparing conscious sedation versus general anesthesia in transcatheter aortic valve implantation. Am J Cardiol 2019;124:70-77.

Nuis RJ, Rodes-Cabau J, Sinning JM, et al. Blood transfusion and the risk of acute kidney injury after transcatheter aortic valve implantation. Circ Cardiovasc Interv 2012;5:680-688.

Oguri A, Yamamoto M, Mouillet G, et al. Clinical outcomes and safety of transfemoral aortic valve implantation under general versus local anesthesia: Subanalysis of the French Aortic National Corevalve and Edwards 2 registry. Circ Cardiovasc Interv 2014;7:602-610.

Saia F, Ciuca C, Taglieri N, et al. Acute kidney injury following transcatheter aortic valve implantation: Incidence, predictors and clinical outcome. Int J Cardiol 2013;168:1034-1040.

Thiele H, Kurz T, Feistritzer HJ, et al. General versus local anesthesia with conscious sedation in transcatheter aortic valve implantation: The randomized SOLVE-TAVI trial. Circulation 2020;142:1437-1447.

Van Mieghem NM, Tchetche D, Chieffo A, et al. Incidence, predictors, and implications of access site complications with transfemoral transcatheter aortic valve implantation. Am J Cardiol 2012;110:1361-1367. 
Villareal RP, Hariharan R, Liu BC, et al. Postoperative atrial fibrillation and mortality after coronary artery bypass surgery. J Am Coll Cardiol 2004;43:742-748.

Yagasaki H, Goto Y, Mori Y, Noda T. Transcatheter aortic valve replacement with intracardiac echocardiography from the right internal jugular vein. Cardiovasc Diagn Ther 2018;8:525-529.

Zaouter C, Smaili S, Leroux L, et al. Transcatheter aortic valve implantation: General anesthesia using transesophageal echocardiography does not decrease the incidence of paravalvular leaks compared to sedation alone. Ann Card Anaesth 2018;21:277-284. 